livraisons

d'Histoire

de l'Architecture

\section{Livraisons de l'histoire de l'architecture}

30 | 2015

Le dessin d'architecture : œuvre/outil des architectes

?

\title{
Dessins et design : représenter l'espace mental de l'architecture : les dessins déconstructivistes de Daniel Libeskind : Micromegas
}

Drawings and design: The representation of mental space in architecture:

Drawings by Libeskind: Micromegas

Zeichnung und Design: den mentalen Raum der Architektur wiedergeben:

Zeichnungen von Daniel Libeskind: Micromegas

\section{Antonella Tufano}

\section{(2) OpenEdition}

Journals

Édition électronique

URL : http://journals.openedition.org/lha/553

DOI : 10.4000//ha.553

ISSN : 1960-5994

Éditeur

Association Livraisons d'histoire de l'architecture - LHA

Édition imprimée

Date de publication : 30 décembre 2015

Pagination : 81-92

ISSN : 1627-4970

\section{Référence électronique}

Antonella Tufano, «Dessins et design : représenter l'espace mental de l'architecture : les dessins

déconstructivistes de Daniel Libeskind: Micromegas », Livraisons de l'histoire de l'architecture [En ligne], 30 | 2015, mis en ligne le 18 décembre 2017, consulté le 04 juin 2020. URL : http://

journals.openedition.org/lha/553; DOI : https://doi.org/10.4000//ha.553 


\section{DESSINS ET DESIGN : REPRÉSENTER L'ESPACE MENTAL DE L'ARCHITECTURE : LES DESSINS DÉCONSTRUCTIVISTES DE DANIEL LIBESKIND : MICROMEGAS}

Les dessins d'architecture sont les porteurs d'une poétique et d'une esthétique de l'espace et, souvent, ils racontent une vision du monde, une projection, que l'architecte concrétise dans ses traits.

Au tournant des années 1970-1980, le dessin d'architecture se réinvente sous l'effet de facteurs esthétiques, techniques, économique et politiques au cœur de la conception et réalisation des architectures et des villes. Ce tournant opère une mutation importante non seulement dans la représentation, mais également dans la manière de concevoir l'espace.

Les dessins postmodernes ou déconstructivistes racontent cette métamorphose et sont les indicateurs de la genèse d'un nouvel espace mental du design, en entendant par design la démarche de conception globale nécessaire pour des projets aussi différents qu'un objet ou une ville.

Cette métamorphose se déroule en trois temps que les dessins de Daniel Libeskind synthétisent : le dessin se transforme d'un outil de représentation à un outil de présentation d'un espace architectural d'une nature nouvelle.

Depuis la Renaissance, la représentation architecturale crée un espace mental pour une architecture virtuelle; cette construction correspond à une modification de la manière d'entendre le rôle de l'architecte. Ainsi, lorsqu'au tournant des années 1970 le numérique impose un espace virtuel qui simule l'espace réel, la construction moderne et rationnelle est soumise à une observation par ce nouveau prisme.

À son tour, la simulation du réel impose une ré-réflexion esthétique du dessin et amorce une deuxième révolution. Elle intervient en parallèle avec de profondes métamorphoses sociétales et pose une question cruciale : à quoi sert le dessin à l'âge de la simulation numérique? Au lieu de le rejeter ou de le transformer en acte esthétique disjoint du projet, le dessin se réinvente grâce au travail critique de certains architectes. Il devient le vecteur de monstration d'une rupture radicale dans la manière de concevoir les espaces et les codes architecturaux.

Cette radicalité se transforme en outil épistémologique : le dessin peut être décodé et donne à lire la mutation du travail de l'architecte; la feuille blanche s'ouvre sur un espace sans fonds, devient la projection de l'espace mental de l'architecture. La lecture de Micromegas, dessins réalisés par Daniel Libeskind en 1979, montre leur capacité à donner à voir cet espace mental ainsi que le cheminement de rupture et la réinvention du code architectural. 


\section{Représenter un espace autre}

Nul doute qu'en architecture le dessin est avant tout la projection d'une idée, un projet dont les formes sont multiples, comme le rappelle le Thesaurus de la langue française.

Dessin et projet se lient pour devenir à la fois un message, une forme et un contenu. C'est une pratique ancienne dont la codification géométrique intervient à la Renaissance. Avec la mise en perspective géométrique, sont posées les conditions pour légitimer un espace parallèle où le projet dessiné possède une force inégalée. Il ne s'agit pas simplement de l'expédient pour représenter l'espace et y déposer de manière ordonnée les objets, mais d'un espace en soi où l'on peut indifféremment poser des objets représentés ou des objets inventés.

Comme le note Franco Alessio ${ }^{1}$, la perspective existait depuis le XIII ${ }^{\mathrm{e}}$ siècle comme instrument - un peu technique et un peu théologique - pour comprendre la lumière et ses effets.

À la fin du XIII e siècle, d'une part, la perspective glisse de l'étude de la métaphysique à celui de la physique en devenant un instrument optique et, d'autre part, fait sortir la lumière du registre surnaturel pour la transformer échelon de mesure de la profondeur de l'espace. À travers la médiation critique de Biagio Palacani de Parme $^{2}$, Brunelleschi effectue une synthèse entre la perspective et les différents objets représentés - ville, architecture, territoire - qui restera le code de représentation le plus utilisé pendant des siècles. Les traités d'architecture diffuseront et vulgariseront cette complexe construction, où la représentation correspond à une réalité d'une autre nature. Comme le résume Ciucci : "L'œil de Ledoux qui reflète la réalité semble s'identifier à ce "Je" (celui de Descartes) : la lumière qu'illumine le monde physique de l'architecture, l'espace artificiel, semble avoir origine dans l'esprit; l'unité n'est plus dans la nature, naturelle ou artificielle, mais bien dans l'esprit de l'homme ${ }^{3}$ " et les instruments de représentation comme la géométrie descriptive sont : "le moyen pour chercher la vérité ». Werner Oechslin montre dans ses ouvrages la légitimation que les mathématiques offrent à cet espace architectural créé par l'esprit, un espace à la fois lisible et abstrait.

Système spatial indépendant, le dessin subit une transformation centrale avec sa virtualisation et la simulation des espaces que les outils numériques rendent possible dans les années 1970. À partir des premières expériences de Muriel Cooper ${ }^{4}$ au MIT, en 1973, notamment l'expérience Visible Language Workshop, un autre espace, dit « cyberespace ", s'oppose à l'espace construit géométriquement. En effet, l'image figurée, réplique du réel, saturée de messages univoques, bouscule les référentiels classiques, tant dans la conception que dans la réalisation des projets ; ainsi,

1. Franco Alessio, La filosofia e le artes mechanicae, Spoleto, CISAM, 1984, 160 p.

2. Graziella Federici Vescovini, Filosofia, scienza e astrologia nel Trecent europeo, Padoue, Il Poligrafo, 1992, 222 p.

3. Franco Ciucci, "Rappresentazioni ", Rassegna, n 9, Bologne, CIPIA, 1982, p. 11.

4. Muriel Cooper participe avec Nicolas Negroponte à la mise en place du Media Lab du MIT, dont son atelier était une préfiguration. 
après l'épopée moderne qui avait porté à ses extrêmes les possibilités classiques du dessin et assuré à l'architecte un rôle de démiurge, la crise de la modernité rend nécessaire une réflexion où la place du dessin est, en apparence, mise à mal par la force du virtuel.

La virtualisation de l'espace est un phénomène poussant l'abstraction à ses limites ou, comme le précise Galimberti ${ }^{5}$, accomplissant la tâche conjointe de la philosophie et de la mathématique qui encouragent à formuler des concepts de référence pour toutes les formes du concret. Depuis Descartes l'esprit a le rôle de construire une réalité virtuelle, de discerner "dans une image dématérialisée, l'effet de la réalité matérielle sans l'encombre de la matière ${ }^{6}$; ; avec la critique de la modernité, l'esprit est soumis à une critique dont le but est la compréhension du nouveau statut de la forme dématérialisée. Une forme dématérialisée n'assumant plus l'abstraction mais se proposant comme un alter-espace qui, paradoxalement, réduit les possibilités d'interprétation et limite radicalement son potentiel d'exploration.

\section{Espace postmoderne et déconstructiviste : la mutation du rôle du dessin}

La virtualisation et la dématérialisation de l'image sont les deux aspects les plus visibles de la virtuosité atteinte par les représentations numériques; cette prouesse technique, très versatile, se prête à toutes les interprétations, ainsi l'image hyper réelle est au centre des deux courants qui dominent les années 1970-1980 : le postmoderne et la déconstruction. Les deux mouvements partagent la volonté de sortir de la modernité ; les deux accordent à l'image une place prépondérante et, pour s'opposer à une image médiatique trop écrasante, conferent au dessin un pouvoir de critique.

La postmodernité critique va plus loin que le retour au passé utilisé par les architectes $^{7}$. Elle se fonde sur des contradictions et paradoxes que le critique littéraire Ihab Hassan ${ }^{8}$ avait remarquablement identifiés à travers des oppositions, dont les plus importantes sont celle du logos au silence, de la présence à l'absence, de l'enracinement au rhizome, de la narration à la micrologie ou encore de l'origine à la différence.

5. Umberto Galimbert, Parole nomadi, Milan, Universale Feltrinelli, 2006 (1994), 242 p.

6. Umberto Galimberti, Parole nomadi, op. cit., p. 221.

7. Charles Jencks, The Post-Modern Reader, Londres, Academy editions, 1992, 416 p. Le débat entre les postmodernes, tel que Jencks, qui reprochent à la déconstruction son nihilisme, donnera lieu à une vaste littérature, dont la réponse la plus tranchante est donnée par Jacques Derrida dans un entretien avec Christopher Norris, "Jacques Derrida in discussion with Christopher Norris", Andreas Papadakis, Catherine Cooke et Andrew Benjamin, Deconstruction. Omnibus Volume, Londres, Academy Editions, 1989, p. 70 et suiv.

8. Hassan Ihab, "Toward a concept of postmodernism ", The postmodern turn: essays in postmodern theory and culture, Columbus, Ohio State University Press, 1987, p. 84-96. L'article est repris dans l'ouvrage Linda Hutcheon et Joseph Natoli, A Post modern reader, New York, State University of New York Press, 1993, p. 273-286, qui constitue une excellente synthèse des mutations intervenues depuis les années 1970 . 
Dans le droit fil de cette décomposition, le mouvement déconstructiviste sera une méditation sur la dissolution de ces quelques préceptes et concepts. Dans ce contexte, la postmodernité et le déconstructivisme ${ }^{9}$ sont les deux visages d'une même crise de l'architecture et de sa représentation. Les témoignages de deux architectes aussi opposés que Stanley Tigerman et Bernard Tschumi en sont la preuve.

Le premier, formé à Chicago, radicalement investi dans la postmodernité venturienne, souligne le rôle du dessin au tournant des années 1970 comme un élément qui va au-delà de l'aide à la réalisation ou à la compréhension du projet.

Après le choc pétrolier, la construction ralentit; une sensibilité au contexte émerge ; les architectes, redécouvrent alors le dessin comme " consolation et inspiration "; Tigerman affirmera même que la vision conceptuelle ne peut être "nourrie que par la liberté du dessin. ${ }^{10}$ " La Biennale de Venise de 1976 marquera un tournant en montrant l'architecture comme une discipline dont la légitimation est faite par les projets dessinés plus que par les projets réalisés ${ }^{11}$. Comme le disait avec un certain humour le critique italien Giulio Carlo Argan, les dessins sont des d'exercices de gymnastique intellectuelle pour les architectes.

Le deuxième, formé dans les années 1970 à la AA school à Londres, le lieu où le changement se préparait et où se croisent Zenghelis, Koolhaas, Krier et Libeskind, raconte le contexte d'émergence de cette nouvelle vague :

« La France et l'Italie avaient été dévastées par la rupture de 1968 : il n’y avait pas de production, aucun travail. Les Ecoles étaient complémentent épuisées. À cette époque, en Europe, le place to be était vraiment Londres, et AA était le centre de la scène londonienne. [...] Une nouvelle sensibilité architecturale se développait. [...] à cette époque il existait le pressentiment qu'une transformation était en train de s'accomplir. Mais ça sera seulement dix ans après que les personnes commenceront à construire vraiment ${ }^{12}$. "

Et, dans ce laps de temps, les architectes dessinent. Tschumi invente, en 1976, avec le Joyce's garden, un langage nouveau fait de dessin, de textes, de représentations fantasques à la présentation géométrique impeccable.

L'exposition qui s'ouvre au Moma en juin 1988, présentée souvent comme le coup d'envoi de la Deconstructivist Architecture, n'est en fait que le point final de tous ces débats. Les commissaires, l'architecte Philip Johnson et le théoricien Marc Wigley, ne se trompent pas en soulignant le "pluralisme qui règne" ainsi que le

9. Cf. Martin McQuillan, Deconstruction. A reader, New York, Routledge, 2001, 553 p.

10. Cf. l'article intitulé "Architecture and its trace" (1978), Stanley Tigerman, Schlepping through Ambivalence, Londres et New Haven, Yale University Press, 2011, p. 24 et suiv.

11. Intitulée Art/Architecture Year zero, la Biennale de 1976, guidée par Vittorio Gregotti avec l'aide de l'historien Franco Raggi et du critique Germano Celant, avait l'objectif d'élargir la notion d'art à celle d'environnement et d'inclure l'architecture (représentée par une confrontation d'architectes américains et européens), ainsi que le design, à travers l'histoire du Werkbund et l'hommage à Ettore Sottsass.

12. Bernard Tschumi, «Bernard Tschumi ", GA extra, no 10, Tokyo, A.D.A., 1997, p. 16. 
fait que les sept architectes choisis ne forment pas un "isme ${ }^{13}$ " mais sont unis par une volonté de rompre avec les règles très conservatrices de l'architecture, qui est à la recherche depuis des décennies de la forme pure : ils ont en commun de vouloir la déstabiliser ${ }^{14}$.

Le dessin, outil principal de l'architecte, deviendra dans leurs mains l'instrument d'invention de ce nouvel espace. Dans un écrit de 1985 qui accompagne la publication en France du travail dessiné de quelques architectes (Libeskind, Hejduk, Eisenmann...), Jacques Guillerme ${ }^{15}$ a bien mis en évidence la relation entre les dessins de ces architectes (qu'on n'appelle pas encore déconstructivistes) et la révolution informatique. Il y voit un point en commun : la disparition du projet en tant que geste dessiné et, à la place, l'apparition d'un code d'exécution de tâches complexes, un protocole, qui peut se passer de représentation. Devant cette apparente impasse, il s'appuie sur les considérations d'un spécialiste du numérique, Philippe Queau, et affirme qu'on peut enfin faire des projets sans plans. Bien audelà de l'approche simpliste, Guillerme oppose à l'ordinateur en tant que «truchement productif d'un artifice ${ }^{16}$ " la capacité implicite de l'outil à créer une maquette totale, une chose composite faite de visuel, de technique, de numérique, ainsi que de virtuel informatif. La maquette de synthèse est " une gerbe de solutions approximatives ", une image absolue au sens de Bachelard, la préfiguration de celle qu'on appellera plus tard une image paramétrique. Souple et évolutive, cette manière de concevoir permettra d'appréhender le non mesurable, d'ouvrir à la perception.

\section{La résistance par le dessin: Micromegas}

Ce paradoxe, représenter le non représentable, sera donc l'objectif commun des représentations (dessins, photomontages, installations...) déconstructivistes. La transformation de l'architecture en en image de l'architecture et les questions que cela provoque sont le point qui rapproche les démarches utopistes de Coop Himmelblau, à la médiatisation de Koolhaas ou encore à l'expression sculpturale de l'architecture de Gehry. Les explorations numériques conduiront Tschumi et Hadid à des performances dans la conception assistée par ordinateur, qui renverse la relation au dessin traditionnelle. Et, sur un autre versant, l'image accompagnée d'une lecture critique dont le dessin est l'expression sera le dénominateur commun au travail de Peter Eisenmann et Daniel Libeskind, ces derniers ayant partagé aussi l'expérience de pédagogie et de recherche au sein de Cooper Union avec John Hejduk ${ }^{17}$.

13. Coop Himmelblau, Eisenmann, Gehry, Hadid, Koolhas, Libeskind, Tschumi sont issus de différentes cultures, comme le souligne Johnson, tout en précisant qu'à l'instant même où l'exposition se déroule leur production converge vers un point commun. Cf. Philip Johnson, "Preface ", Deconstructivist Architecture, New York, Moma, 1988, p. 8.

14. Mark Wigley, "Deconstructivist Architecture ", Deconstructivist Architecture, op. cit., p. 10 et suiv.

15. Jacques Guillerme, "Tracés contemporains et espace moderne. La puissance du factice du lavis aux images de synthèse ", Techniques \& Architecture, ${ }^{\circ}$ 358, Paris, Altedia, p. 54-57.

16. Cité par Jacques Guillerme, op. cit., p. 56.

17. Ce dernier ne sera pas exposé au Moma mais gardera une place centrale dans la réflexion déconstructiviste. 
Daniel Libeskind, plus particulièrement, opte pour un dessin qui exprime la "rematérialisation " de l'architecture post-déconstructiviste. Les dessins qu'il réalise dans la période d'enseignement à Cranbrook, entre 1978 et 1985, portent in nuce le déploiement possible de son architecture.

Par le dessin, il pense un code de projet qui correspond à la refonte totale des outils de conception, de représentation, mais aussi de réalisation de l'architecture. En allant dans une direction plus théorique que les autres, il comprend que la base du problème est la construction perspective renaissante, son illusion de connaissance de la réalité confortée par les formules géométriques.

Il va ainsi proposer une lecture alternative, méticuleusement étudiée, calculée sur la base d'une interprétation des nombres entendus comme la clé d'un système harmonique emprunté à la musique, qu'il complexifie jusqu'à donner naissance à un univers algorithmique.

Dans un texte manifeste, datant de 1981, Daniel Libeskind revendique : «Ce qui nous intéresse, de toute manière, est la dimension herméneutique de l'architecture, c'est-à-dire la tentative de récupérer la signification; cette récupération n'est possible que par la concrétisation des objets ${ }^{18}$ ». Une concrétisation des objets, et non une réalisation : ce passage important souligne l'importance accordée au dessin, le seul outil capable de concrétiser un langage alternatif de l'architecture.

Libeskind propose des architectures dessinées qui incorporent l'esprit pour échapper à une réduction de l'architecture au jeu de références ou à l'imitation d'une figure organique. Loin du leurre de l'image augmentée, d'une nature artificielle plus vraie que nature, il insiste sur le fait que le langage architectural est antinaturel et symbolique. C'est un acte d'entendement immédiat, une phénoménologie architecturale qui échappe aux longs procédés de calcul.

Les dessins de Micromegas, réalisés à cette époque, sont le reflet de recherche ; comme le dira Hejduk dans Crossover ${ }^{19}$, il ne s'agit pas des dessins "préparatoires " mais d'œuvres architecturales avec leurs règles de lisibilité et de réception, des dessins qui se légitiment et légitiment leur propre possibilité d'exister. (Ill. 1 à 3) Libeskind parachève la réflexion critique d'un Hejduk sur le dessin, comme le résume Alain Pélissier :

"Mondialement connus par leurs dessins, ils (Hejduk et Libeskind) refusent à ce dernier la primauté sur le bâti. Dessiner, c'est pour eux condenser une somme d'opérations mentales et physiques dont le projet architectural s'alimente également. Dessiner, c'est l'activité synthétique où le projet se met en place. Dessiner, c'est le premier acte constructeur. Construire c'est un acte constructeur d'un autre type. Dessiner et construire participent de la même volonté d'élaborer un objet réfléchi qui ne se contente pas d'être là mais qui donne à penser ${ }^{20}$. "

18. Daniel Libeskind, "La decostruzione dell'architettura ", Lotus International, $\mathrm{n}^{\circ}$ 32, Milan, Electa, 1981, p. 94

19. Ce texte, écrit en décembre 1979, est reproduit dans le catalogue du musée d'Architecture de Helsinki. John Hejduk, "Crossover", Symbol and Interpretation. Cranbrook Academy of Art Department of Architecture, 1981-1982, Helsinki, Museum of Finnish Architecture Press, 1982, p. 58.

20. Alain Pellisier, "L'architecture aux limites ", Machines d'architecture, Paris, Fondation Cartier pour l'Art Contemporain, 1993, p. 11. 


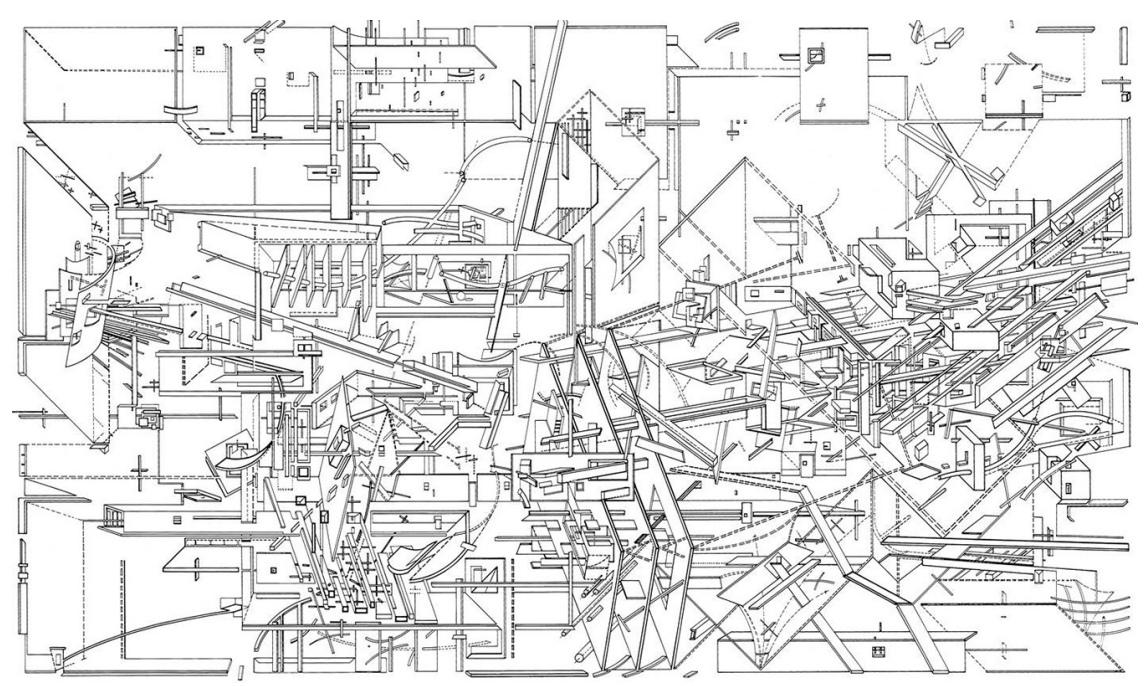

Ill. 1 : Daniel Libeskind, Micromegas, Time Sections, 1979. (C) Courtesy Studio Libeskind.

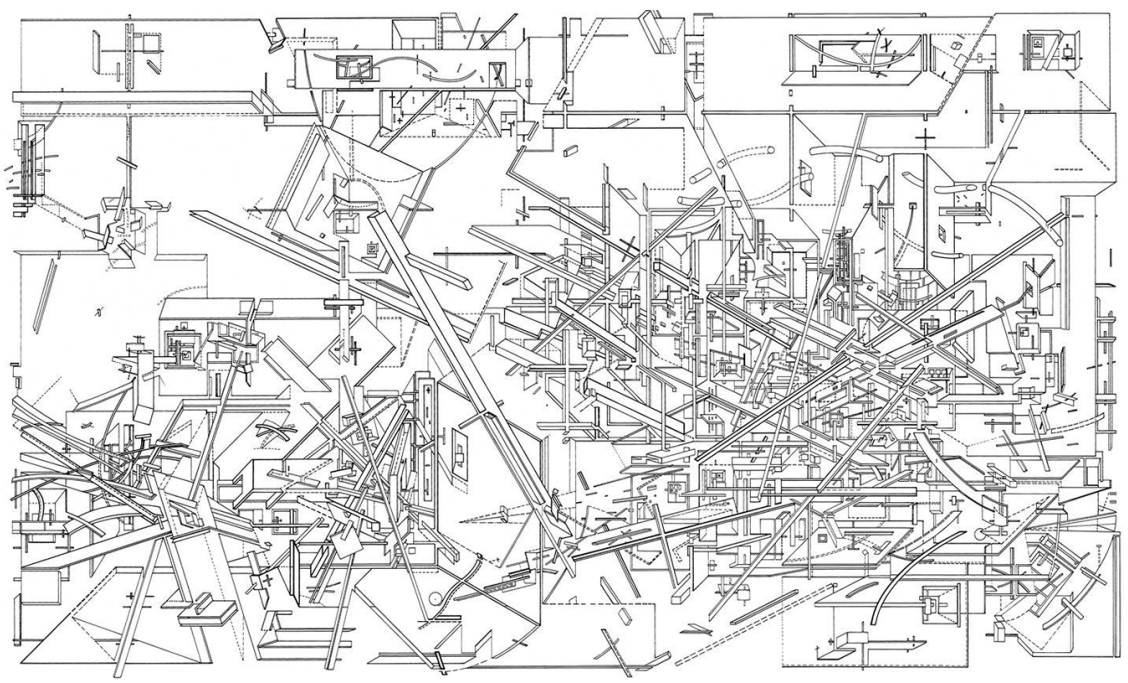

Ill. 2 : Daniel Libeskind, Micromegas, Leakage, 1979. (C) Courtesy Studio Libeskind. 


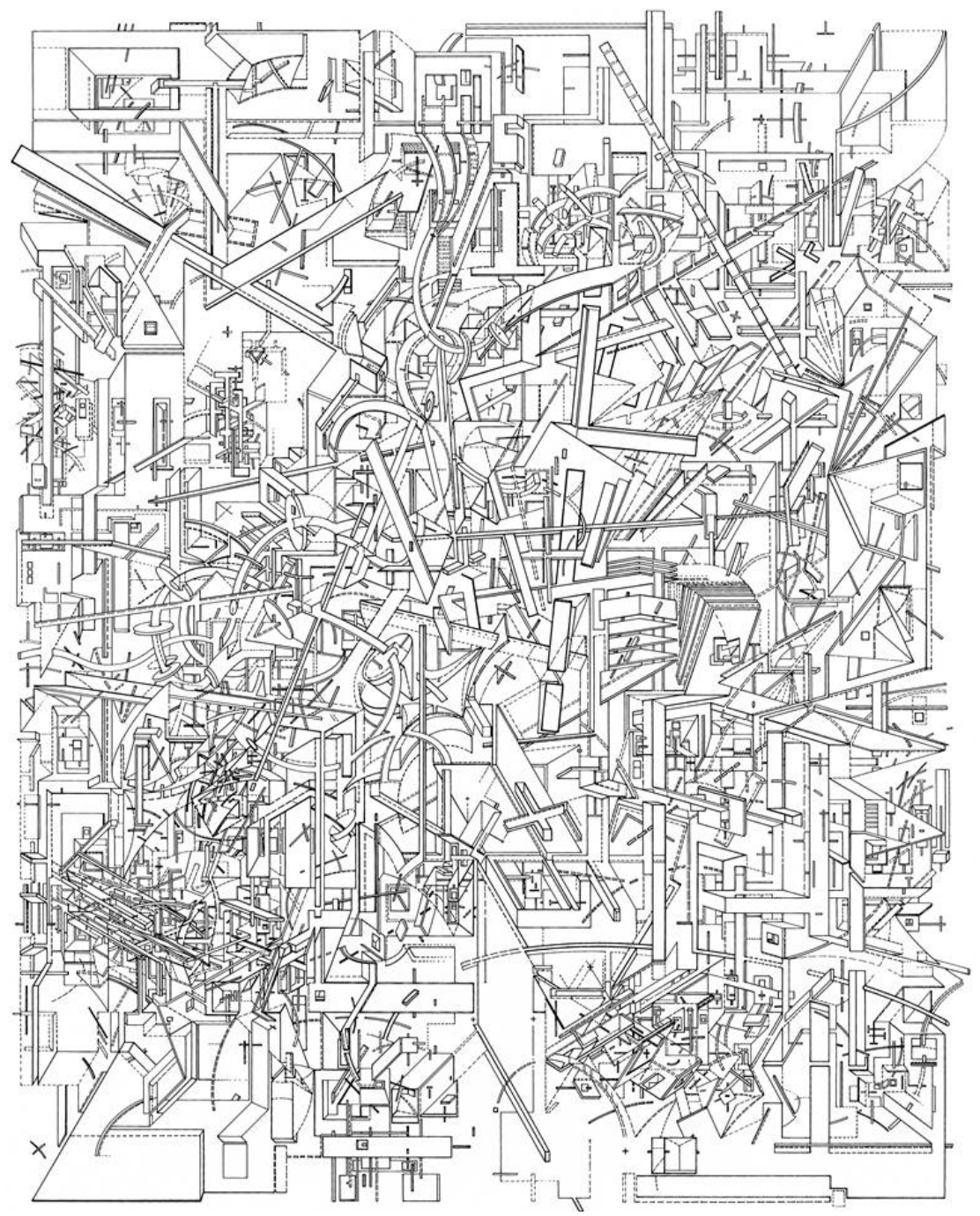

Ill. 3 : Daniel Libeskind, Micromegas, Vertical Horizon, 1979. (c) Courtesy Studio Libeskind.

Daniel Libeskind, architecte aux cultures multiples ${ }^{21}$, ouvert à l'approche sensible, engagé dans l'enseignement avec une grande intensité à la Cranbrook Academy, accomplit donc un acte fondateur de déconstruction et reconstruction avec cette série de dessins.

21. Daniel Libeskind commence ses études de musique en Isräel et les poursuit aux États-Unis. En 1960, il se réoriente vers l'architecture qu'il étudie à Cooper Union où il obtient son bachelor degree avant son master en Histoire et théorie de l'architecture à l'université d'Essex, en Angleterre. Il sera Unit master à la AA, entre 1975 et 1977, et devient chef du département d'architecture à Cranbrook en 1978. 
Exposés à Helsinki ${ }^{22}$, puis dans le reste de l'Europe lors de la célébration des cinquante ans d'activités de la Cranbrook Academy of Art, ces dessins font aussi l'objet de l'admiration de l'architecte commissaire de l'exposition, Juhani Pallasmaa ${ }^{23}$. Pallasmaa souligne la précision presque maniaque que Libeskind met à réaliser ses dessins, comme s'ils devaient se matérialiser et concrétiser cette nouvelle architecture où se croisent :

«Secret scores of astronomical rites;

El Lisstzky's Prouns to second power;

Encyclopedia of architectural motives;

Sound clusters of Ligeti and Penderetski in visual form ${ }^{24}$."

Présenté dans un coffret en nombre limité, Micromegas rend hommage à Voltaire non pas comme une illustration du conte philosophique, mais en tant qu'invention d'une narration architecturale qui n'a ni début ni fin, où les échelles s'entrelacent, une narration qui déconstruit ironiquement les éléments de l'histoire de la modernité. "L'espace de l'architecture dessinée ne connaît aucune restriction ou gravité ", dira encore Pallasmaa, le dessin n'est plus «simplement une manière de communiquer des images architecturales, le dessin est une pensée architecturale ${ }^{25}$. "

Dans Micromégas, Voltaire raconte le voyage d'initiation de son protagoniste - un savant venant de Syrius, à la taille impressionnante de trente kilomètres accompagné d'un savant nain - qui ne mesure que quelques dizaines de kilomètres. Les deux voyageurs se penchent sur le sort des habitants de plusieurs planètes, jusqu'à une étrange planète, la Terre, où des minuscules habitants ont la présomption de tout connaître. Récit à interprétations multiples, Micromégas est une critique portée à la science lorsqu'elle s'arroge le droit de donner accès à la Vérité. En pensant par le dessin, le Micromegas de Libeskind, propose des scènes à interprétation multiple où commence le redéploiement de l'architecture: The Garden, Time sections, Leakage, Little Universe, Artic flowers, The burrow laws, Dance sounds, Maldoror's equation, Vertical horizon, Dream Calculus, sont en effet, des micrologies que l'architecte nous livre presque sans commentaires.

Le voyage de Daniel Libeskind commence dans un jardin, The Garden, qui donne vie à une "explosion sans bruit ${ }^{26}$. " Cette explosion marque la genèse d'une nouvelle architecture qui ne peut pas se lier à une quelconque tradition : "Notre architecture est toujours dans le présent ; il n'existe plus d'âge ancien, dans le jardin il n'y a plus d'arbres ou de chlorophylle, on se meut d'obscurité en obscurité. Nous

22. Cranbrook a été modelé par Eliel Saarinen dont l'enseignement reste encore une référence dans la culture finlandaise, notamment dans la génération de Pallasmaa par la médiation de Gullichsen.

23. Daniel Libeskind, Micromegas. Architectural drawings, Helsinki, Artek, Museum of Finnish Architecture, 1980, $20 \mathrm{p}$.

24. Juhani Pallasmaa, " 3 Images in the Libeskind macroscope ", Micromegas, op. cit., p. 5.

25. Juhani Pallasmaa, "The Architectural Drawing - Tool and end Product", Micromegas, op. cit., p. 10 et suiv.

26. Cf. ci-dessus John Hejduk, "Crossover ", op. cit., p. 58 
sommes les témoins d'un épisode de naissance de l'architecture ${ }^{27}$. " De ce point de non-origine, commence une exploration dont le but est de détruire quelques fausses certitudes architecturales.

\section{Détruire la surface}

Libeskind intitulera un texte de la fin des années 1980 The Surface Must Die. A Proof ${ }^{8}$, qui explique non seulement la nécessité de déstructurer la forme, mais aussi de réinventer un langage. Le temps semble donc exploser dans le dessin Time sections et un ironique point d'horizon ou fuite est proposé par Leakeage.

\section{Détruire la fausse représentation naturaliste}

Artic Flowers et Little Univers se répondent dans l'emboîtement du grand et du petit, une superposition entre les rythmes temporels. Il s'agit peut-être des dessins plus proches du texte de Voltaire, notamment dans les passages où l'observation au microscope jette le savant Micromégas dans le plus grand désarroi et l'amène à conclure que l'observation sans esprit ne sert à rien, qu'il faut aller au-delà du sens commun et se fier à la perception.

\section{Détruire le sens commun}

Les dessins The burrow laws et Maldoror's equation renvoient au rôle de l'imagination comme faculté qui dépasse tout naturalisme. Mise en abîme d'autres textes littéraires, notamment le Terrier de Kafka et les Chants de Maldoror, cet ensemble de dessins exprime une fragmentation surréaliste où l'incomplétude des figures accompagne le vacillement des certitudes.

\section{Détruire toute certitude}

Le dessin Sound dances fonctionne comme un pivot. Dans cet univers sans sons ni bruits, s'ouvre un probable espace immatériel, où le chiffre ne compose pas de vertigineuses constructions rationnelles mais donne des indications subjectives à suivre. ${ }^{29}$ Une orientation relative dans une géométrie éclatée.

\section{Détruire la géométrie}

Libeskind affirme avoir commencé les dessins en lisant les Origines de la géométrie de Husserl ${ }^{30}$. Vertical horizon ${ }^{31}$ est un dessin qui pose donc frontalement la

27. John Hejduk, op. cit., p. 58.

28. Daniel Libeskind, "The surface must die ", AD Deconstruction II n ${ }^{\circ}$ 77, Londres, Academy Press, 1989 , p. 21

29. Thorleif Boman, Hebrew Thought compared with Greek, Philadelphie, Westminster Press, 1960, $224 \mathrm{p}$.

30. Le texte de Husserl est écrit en 1936 et publié en allemand en 1954 ; il paraît en France dans une édition traduite et introduite par Jacques Derrida : Edmund Husserl, L'origine de la géométrie, Paris, PUF, 1962, 219 p.

31. Ce thème sera poursuivi dans une autre série de dessins; cf. Daniel Libeskind, Chamber Works : Architectural meditations on Themes from Heraclitus, Londres, Architectural Association, 1983, 33 p. 
question des repères spatiaux et Dream Calculus termine la série avec une redoutable désintégration de toutes les composantes spatiales. Comme il l'écrit :

« [En] comprenant que la genèse historique de la géométrie a subi une évolution à partir des problèmes liés à la mesure du réel (j’entends le calcul comme produit de l'étude du mouvement ou les statistiques comme produit de l'étude des collectivités), j'ai compris de mieux en mieux que l'ouverture du premier horizon (en délimitant par-là l'espace des premières découvertes), est le garant du lien dans le projet d'objectivation.»

Il ne s'agit donc pas de superposer des hiérarchies, mais bien de donner des couches d'interprétation différentes : "draws only which allows itself to be drawn into $^{32}$ ", incite Libeskind en revendiquant pour ses dessins un statut "[d']énigmes [riddles], instruments inconnus dont l'usage reste encore à trouver ${ }^{33}$.»

\section{De Venise à Venise...}

Ses conclusions sibyllines renvoient à l'œuvre de Voltaire. Le philosophe termine en racontant comment Micromégas

«Promit [aux humains] de leur faire un beau Livre de Philosophie, qui leur apprendroit des choses admirables \& qui leur montreroit le bon des choses. Effectivement il leur donna ce Livre avant son départ : on le porta à Paris à l'Académie des Sciences; mais quand le vieux Secrétaire l'eut ouvert il ne vit rien qu'un Livre tout blanc. Ah! dit-il, je m'en étois bien douté ${ }^{34}$.»

À cette conclusion ouverte, Libeskind répond par une pratique du dessin de plus en plus nourrie d'éléments classiques et, en même temps, de plus en plus déstructurante. L'acte ultime de cette phase, seront les machines qu'il réalise en 1985.

En 1985, Libeskind propose pour la Biennale de Venise une installation intitulée Three lessons in Architecture, composée de trois machines inspirées de textes et concepts anciens: Reading Machine, Writing Machine et Memory Machine ${ }^{35}$. Il va recevoir la médaille de la Biennale et entamer le chemin de constructeur qui le conduit à Berlin où il accompli avec Between the lines son projet phare pour l'extension du Musée de culture juive.

En 2014, Daniel Libeskind revient à la Biennale de Venise intitulée Fundamentals (ill. 4). Dans cette apothéose de la pensée koolhaassienne, il crée un espace à part,

32. Ce texte accompagne les dessins de Micromegas et il est reproduit dans la monographie sur Libeskind, AD Countersign, Architectural monograph, no 16, Londres, Academy Press, p. 10 et suiv.

33. Ibid., p. 15 et suiv.

34. Voltaire, Micromégas (Le) de M. Voltaire, Londres, s. e., 1752, p. 92-93.

35. Ces machines seront exposées à Paris en 1992. Cf. Daniel Libeskind, "Sens dessus dessous ", Machines d'architecture, op. cit., p. 23. 


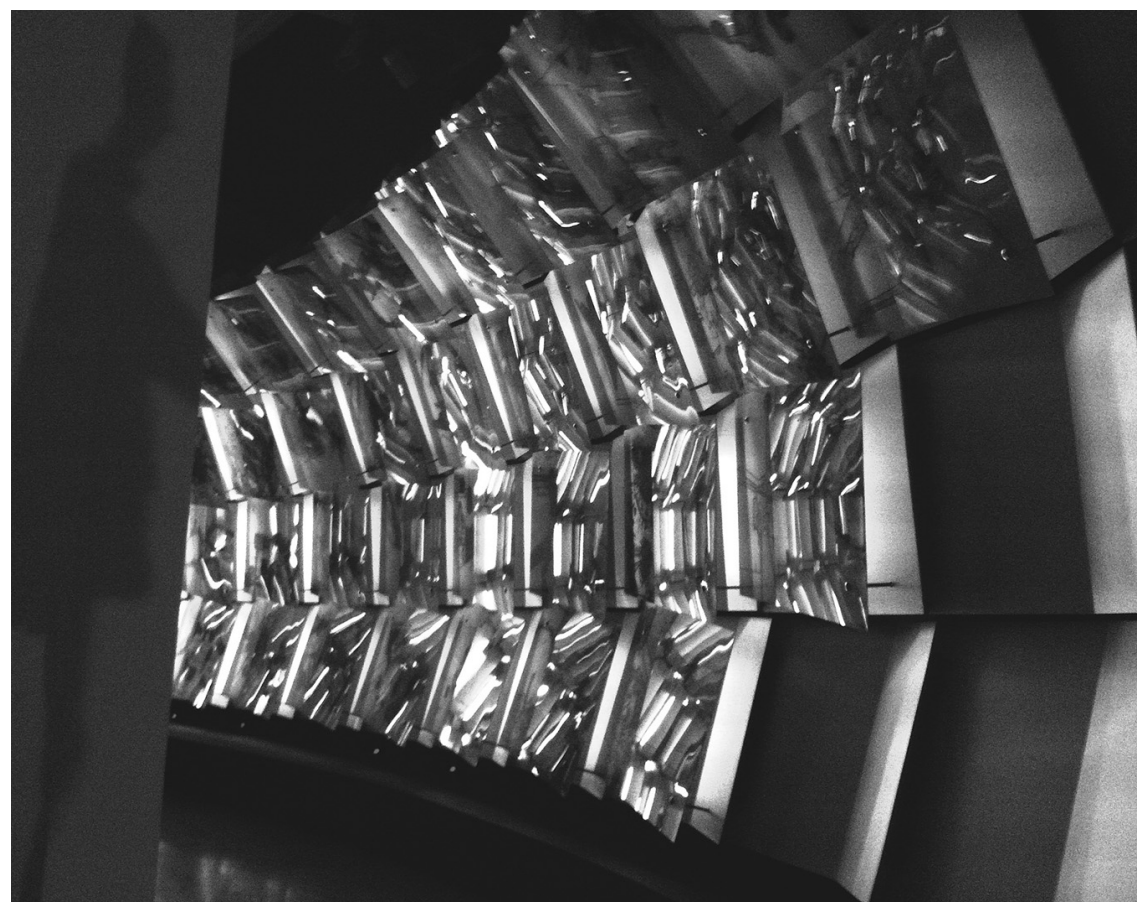

Ill. 4 : Daniel Libeskind, Sonnets in Babylon, 2014, Biennale de Venise, Italie. (C) Cliché Antonella Tufano.

un espace en abîme, où le dessin se matérialise en un couloir lumineux. Intitulé Sonnets in Babylon, cet ensemble de dessins représente des formes non identifiées, naturelles et artificielles, des villes et des corps s'entrechoquant sur des surfaces translucides. Agrandis, ces dessins nous font rentrer dans une pénombre qui s'oppose en tout point à l'hypercommunication koolhaassienne. Quelques textes énigmatiques et des sonnets composés par Libeskind accompagnent cette expérience spatiale. Dans un coin inaccessible, en haut, un tableau noir annonce une Recette qui nous laisse espérer dans une clé de lecture que, in fine, il ne donnera pas.

Antonella TUFANO maître assistant à l'ENSAPLV | chercheur au laboratoire Gerphau-Lavue UMR 7218 